\title{
Seletividade de herbicidas aplicados em pré-emergência na cultura do crambe ${ }^{1}$
}

\author{
Selectivity of herbicides sprayed in pre-emergency in crambe crop
}

\author{
Antonio Mendes de Oliveira Neto ${ }^{2}$, Naiara Guerra ${ }^{2}$, Cleber Daniel de Goes Macie ${ }^{3}$, Tiago Roque \\ Benetoli da Silva ${ }^{3}$, Gesley Guimarães de Ramos Lima ${ }^{4}$
}

Resumo - No Brasil não existem herbicidas registrados para o crambe (Crambe abyssinica Hochst), e pouco se sabe sobre a seletividade de herbicidas sobre essa cultura. Portanto, dois ensaios foram conduzidos simultaneamente em estufa plástica, com objetivo de avaliar a seletividade de herbicidas aplicados em pré-emergência no desenvolvimento inicial da cultura do crambe. No primeiro ensaio avaliou-se a dose-resposta dos tratamentos herbicidas trifluralin

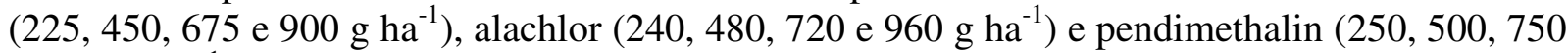
e $1000 \mathrm{~g} \mathrm{ha}^{-1}$ ), em esquema fatorial (3x4)+1, representado pelos fatores herbicidas (três) e doses (quatro), e uma testemunha. No segundo ensaio foram avaliadas quatro misturas em tanque de herbicidas: trifluralin+alachlor $\left(450+480 \mathrm{~g} \mathrm{ha}^{-1}\right)$, trifluralin+pendimethalin $\left(450+500 \mathrm{~g} \mathrm{ha}^{-1}\right) \mathrm{e}$ alachlor+pendimethalin $\left(480+500 \mathrm{~g} \mathrm{ha}^{-1}\right)$ e uma testemunha. Em ambos ensaios utilizou-se vasos com capacidade de $4 \mathrm{dm}^{3}$ de solo, em delineamento inteiramente casualizado, com oito repetições. A cultura do crambe mostrou-se extremamente sensível aos herbicidas pré-emergentes avaliados. Apenas o trifluralin, em doses inferiores a $450 \mathrm{~g} \mathrm{ha}^{-1}$, demonstrou-se seletivo a cultura do crambe. Assim, ficou evidente a necessidade de estudos mais abrangentes que visem avaliar a seletividade de herbicidas à cultura do crambe.

Palavras-chave: Crambe abyssinica, trifluralin, pendimethalin, alachlor.

\begin{abstract}
In Brazil, there are no herbicides registered for crambe (Crambe abyssinica Hochst), and little is known about the selectivity of herbicides over this crop. Therefore, two experiments were conducted, simultaneously, in plastic green house, with the objective of evaluating the selectivity of herbicides sprayed in pre emergency over crambe crop initial development. In the first study, it was evaluated the answer-dose of herbicides treatments trifluralin $(225,450,675$ and $\left.900 \mathrm{~g} \mathrm{ha}^{-1}\right)$, alachlor $\left(240,480,720\right.$ and $\left.960 \mathrm{~g} \mathrm{ha}^{-1}\right)$ and pendimethalin $(250,500,750$ and $\left.1000 \mathrm{~g} \mathrm{ha}^{-1}\right)$, in factorial scheme $(3 \times 4)+1$, represented by herbicides (three) and doses (four) factors and a check. In the second experiment, four herbicides tank mixtures were analyzed: trifluralin + alachlor $\left(450+480 \mathrm{~g} \mathrm{ha}^{-1}\right)$, trifluralin + pendimethalin $\left(450+500 \mathrm{~g} \mathrm{ha}^{-1}\right)$ and alachlor + pendimethalin $\left(480+500 \mathrm{~g} \mathrm{ha}^{-1}\right)$ and a check. In both studies, vases containing $4 \mathrm{dm}^{3}$ of soil were used in randomized entirely design with eight replications. Crambe crop was extremely sensitive

\footnotetext{
${ }^{1}$ Recebido para a publicação em 15/02/2011 e na forma revisada em 29/03/2011.

${ }^{2}$ Eng $^{\mathrm{o}} \mathrm{Agr}^{\mathrm{o}}$, Mestrando do Programa de Pós-graduação em Agronomia da Universidade Estadual de Maringá, NAPD/UEM, Bolsista Capes. E-mail: am.oliveiraneto@ hotmail.com; naiara.guerra@ hotmail.com

${ }^{3}$ Eng $^{\circ}$-Agro ${ }^{\circ}$, D.Sc. Professor Adjunto, Depto de Ciências Agronômicas - Universidade Estadual de Maringá, UEM. Campus Avançado de Umuarama/PR - CAU/CCA. Estrada da Paca s/n, 87507-190, Umuarama/PR E-mail: cdgmaciel2@uem.br; trbsilva@uem.br

${ }^{4}$ Discente do curso de Agronomia da Escola Superior de Agronomia de Paraguaçu Paulista, ESAPP. E-mail: gesley.lima@hotmail.com
} 
to pre emergency herbicides evaluated. Only trifluralin, in doses inferior to $450 \mathrm{~g} \mathrm{ha}^{-1}$, demonstrated selective to crambe crop. Thus, it was evident the need for more comprehensive studies aimed to assessing the selectivity of herbicides to crambe crop.

Key-words: Crambe abyssinica, trifluralin, alachlor, pendimethalin.

O crambe (Crambe abyssinica Hochst. deve ser realizado o controle das plantas ex Fries) é uma planta de ciclo anual, daninhas. pertencente à família das crucíferas, apresenta altura entre 70 e $90 \mathrm{~cm}$, florescimento aos 35 dias após a semeadura, e suas sementes são caracterizadas por serem redondas e revestidas por um tegumento cinza, com 35 a $60 \%$ de óleo. É uma espécie nativa do Mediterrâneo, que vem sendo cultivada em algumas regiões tropicais e subtropicais pelo interesse industrial no óleo extraído de suas sementes, recentemente utilizado para produção de biodiesel (Fundação MS, 2009; Carneiro et al., 2009).

No Brasil, as pesquisas com a cultura do crambe tiveram início em 1995 na Fundação Mato Grosso do Sul, objetivando avaliar o seu comportamento na formação de cobertura do solo (Baez, 2007; Pitol, 2008). No entanto, com o advento da produção de biodiesel essa oleaginosa se tornou opção bastante interessante por apresentar vantagens como precocidade, tolerância à seca e a geadas, baixo custo de produção, produtividade entre 1000 e $1500 \mathrm{~kg} \mathrm{ha}^{-1}$, assim como maior produção de óleo em relação a culturas como girassol, nabo forrageiro, canola, pinhão manso, entre outras. Além disso, o crambe não compete com culturas destinadas à produção de alimento, tornando-se viável o seu cultivo para produção de biocombustível (Baez, 2007; Jasper et al., 2010).

Segundo Endres \& Schatz (1993), o controle de plantas daninhas é um fator crítico para o sistema produtivo do crambe, pois essa cultura não possui grande capacidade de competição com as plantas daninhas durante o desenvolvimento vegetativo inicial. Para ocorrer o fechamento do dossel são necessários de 3 a 4 semanas, sendo esse o período no qual
Os herbicidas trifluralin e pendimethalin têm como mecanismo de ação a inibição da divisão celular, impedindo a polimerização da tubulina, inibindo o crescimento da radícula e a formação de raízes secundárias. Já o alachlor pertence ao grupo químico das cloroacetaminas, o qual ainda não tem mecanismo de ação definido, apesar de existirem relatos de que atuam inibindo a formação de ácidos graxos de cadeia muito longa, além de também poder inibir a síntese de lipídeos, terpenos, flavonóides e proteínas. Tais herbicidas são recomendados para a aplicação em pré-emergência das plantas daninhas e da cultura, pois afeta a divisão celular e como consequência o desenvolvimento da plântula logo após a germinação (Oliveira Jr., 2001; Rodrigues \& Almeida, 2005; Silva et al. 2007).

No Brasil não existem herbicidas registrados para a cultura do crambe, e as estratégias de controle de plantas daninhas precisam ser identificadas para tornar essa cultura viável, portanto realizou-se esse trabalho com o objetivo de avaliar a seletividade de herbicidas aplicados em préemergência no desenvolvimento inicial da cultura do crambe.

Dois experimentos foram conduzidos simultaneamente, entre os meses de julho e agosto de 2008, em estufas plásticas pertencentes à Escola Superior de Agronomia de Paraguaçu Paulista/SP - FUNGE/ESAPP, localizada em altitude média de 506 metros, latitude $22^{\circ} 34^{\prime} 53^{\prime \prime}$ S e longitude $50^{\circ} 34^{\prime} 35^{\prime \prime} \mathrm{W}$.

O solo utilizado nos experimentos foi classificado como Latossolo Vermelho distroférrico (textura arenosa), constituído por 
$803 \mathrm{~g} \mathrm{~kg}^{-1}$ de areia; $122 \mathrm{~g} \mathrm{~kg}^{-1}$ de argila e $75 \mathrm{~g}$ $\mathrm{kg}^{-1}$ de silte. A análise química do solo, em amostras de 0 a $20 \mathrm{~cm}$ de profundidade, revelou $\mathrm{pH}$ de 5,5 $\left(\mathrm{CaCl}_{2}\right) ; 17 \mathrm{mmol}_{\mathrm{c}} \mathrm{dm}^{-3} \mathrm{de}^{+}+\mathrm{Al}^{3+}$; 32,0 $\mathrm{mmol}_{\mathrm{c}} \mathrm{dm}^{-3}$ de $\mathrm{Ca}^{+2} ; 9,0 \mathrm{mmol}_{\mathrm{c}} \mathrm{dm}^{-3} \mathrm{de}$ $\mathrm{Mg}^{+2} ; 1,3 \mathrm{mmol}_{\mathrm{c}} \mathrm{dm}^{-3}$ de $\mathrm{K}^{+} ; 32,0 \mathrm{mg} \mathrm{dm}^{-3}$ de P; 12,0 $\mathrm{g} \mathrm{dm}^{-3}$ de MO; SB de 42,0 $\mathrm{mmol}_{\mathrm{c}} \mathrm{dm}^{-3}$; CTC de 59,0 $\mathrm{mmol}_{\mathrm{c}} \mathrm{dm}^{-3}$ e V\% de 71,0\%.

No primeiro experimento avaliou-se a dose-resposta dos tratamentos herbicidas trifluralin $\left(225,450,675\right.$ e $\left.900 \mathrm{~g} \mathrm{ha}^{-1}\right)$, alachlor $\left(240,480,720\right.$ e $\left.960 \mathrm{~g} \mathrm{ha}^{-1}\right)$ e pendimethalin $\left(250,500,750\right.$ e $\left.1000 \mathrm{~g} \mathrm{ha}^{-1}\right)$, em esquema fatorial $(3 \times 4)+1$, representado pelos fatores herbicida (três) e dose utilizada (quatro), e uma testemunha. No segundo foram avaliados como tratamentos as misturas em tanque de herbicidas, representadas por: trifluralin + alachlor $\left(450+480 \mathrm{~g} \mathrm{ha}^{-1}\right)$, trifluralin + pendimethalin $\left(450+500 \mathrm{~g} \mathrm{ha}^{-1}\right)$ e alachlor + pendimethalin $\left(480+500 \mathrm{~g} \mathrm{ha}^{-1}\right)$ e uma testemunha. Em ambos experimentos utilizouse o delineamento experimental inteiramente casualizado, com oito repetições. As unidades experimentais foram constituídas por vasos de polietileno com capacidade de $4 \mathrm{dm}^{3}$ de solo, onde no dia 15 de julho de 2008 foram uniformemente semeadas a $2 \mathrm{~cm}$ de profundidade 20 sementes da cultivar de crambe FMS Brilhante.

Dois dias após a semeadura do crambe (17/07/2008) foi realizada a aplicação dos herbicidas, utilizando-se de um pulverizador costal pressurizado à $\mathrm{CO}_{2}$, com pressão de 207 $\mathrm{kPa}$ e equipado com duas pontas de jato plano DG 110.02 VS, o que proporcionou volume de calda de $200 \mathrm{~L} \mathrm{ha}^{-1}$. No momento da aplicação os dados climáticos foram temperatura média de $18,5^{\circ} \mathrm{C}$, umidade relativa do ar de $58 \%$ e velocidade dos ventos de $1,9 \mathrm{~km} \mathrm{~h}^{-1}$.

No experimento de dose-resposta foram realizadas avaliações de porcentagem de fitointoxicação das plantas de crambe aos 7, 14, 21 e 28 dias após a aplicação (DAA), através de escala visual, onde $0 \%$ (zero) correspondeu a ausência de injúrias e 100\% a morte das plantas, conforme metodologia descrita por SBCPD (1995), assim como a densidade de plantas por vaso aos 14, 21 e 28 DAA. Para as misturas em tanque avaliou-se apenas a porcentagem de fitointoxicação, de forma semelhante ao experimento de dose-resposta.

Os dados foram submetidos à analise de variância pelo teste F. Para o ensaio de doseresposta as médias foram submetidas a análise de regressão.

Na Figura 1 encontram-se os resultados de dose-resposta referentes à porcentagem de fitointoxicação das plantas de crambe após aplicação dos herbicidas trifluralin, pendimethalin e alachlor. Para o trifluralin notou-se que o comportamento desta variável aos 7, 14 e 21 DAA foram semelhantes, apresentando incremento de 0,014, 0,019 e $0,024 \%$ na fitointoxicação, para cada grama de produto adicionada. Os níveis de intoxicação até os 21 DAA não ultrapassaram $20 \%$, mesmo quando se utilizou $900 \mathrm{~g} \mathrm{ha}^{-1}$ de trifluralin. Porém, aos 28 DAA, observou-se elevado incremento nos níveis de fitointoxicação com o aumento da quantidade de trifluralin aplicada. Doses acima de $450 \mathrm{~g} \mathrm{ha}^{-1}$ proporcionaram fitointoxicação superior a $20 \%$, chegando a causar injúrias irreversíveis ( $\geq 80 \%$ ), quando utilizado $900 \mathrm{~g} \mathrm{ha}^{-1}$.

Essa informação evidencia que a aplicação em pré-emergência de doses iguais e/ou inferiores a $450 \mathrm{~g} \mathrm{ha}^{-1}$ de trifluralin, demonstram comportamento seletivo ao desenvolvimento inicial da cultura do crambe. Esses resultados corroboram com os encontrados em trabalho a campo por Stougaard \& Moomaw (1991), onde também foi caracterizada a tolerância do crambe para aplicação de trifluralin em pré-plantioincorporado (PPI), na dose de $840 \mathrm{~g} \mathrm{ha}^{-1}$, em solos com teor de matéria orgânica de $2,6 \%$ e $3,2 \%$. Assim como também confirmam as pesquisas realizadas por Campbell \& Walton (2008) para o Departamento de Agricultura e 
Alimento do Oeste da Australia (DAFWA), que também relatam viabilidade no uso da trifluralin (Treflan 43\%), em pré-plantioincorporado (PPI) para cultura do crambe.

Para o pendimethalin, verificou-se baixa intoxicação nas plantas de crambe aos 7 e 14 DAA, mesmo quando utilizado $1000 \mathrm{~g} \mathrm{ha}^{-1}$ (Figura 1). No entanto, nas avaliações seguintes, realizadas aos 21 e 28 DAA, os sintomas de injúrias nas plantas foram altamente intensificados, caracterizando o herbicida como não seletivo à espécie estudada. Stougaard \& Moomaw (1991) relataram baixo nível de injúrias visuais para mistura pendimethalin + cinmethylin $(1120+1120 \mathrm{~g}$ $\mathrm{ha}^{-1}$ ), assim não foi constatada diferenças significativas para testemunha capinada, em duas localidades estudadas.

Entre os herbicidas estudados, o alachlor foi o que apresentou os maiores níveis de fitointoxicação inicial, mesmo nas menores doses testadas (Figura 1), sendo que ao contrário do trifluralin e pendimethalin, aos $7 \mathrm{e}$ 14 DAA foram constatados níveis de fitointoxicação superior a $50 \%$, para a maior dose estudada $\left(960 \mathrm{~g} \mathrm{ha}^{-1}\right)$. Aos 21 e 28 DAA, os níveis de injúrias foram ainda mais intensos, superando $80 \%$ de fitointoxicação para doses acima de $480 \mathrm{~g} \mathrm{ha}^{-1}$.

Com relação à densidade de plantas (Figura 2), as aplicações de trifluralin, pendimethalin e alachlor promoveram redução na emergência das plantas de crambe, mesmo para as menores doses de 225; 250 e $240 \mathrm{~g} \mathrm{ha}^{-1}$, respectivamente. Entretanto, para esse parâmetro, $450 \mathrm{~g} \mathrm{ha}^{-1}$ de trifluralin também se destacou por causar os menores decréscimos na emergência do crambe.

O aumento na concentração de trifluralin promoveu taxa de decréscimo no número de plantas emergidas de 0,0047 e 0,0107, respectivamente, aos 21 e 28 DAA, para cada grama de herbicida utilizada. Já com pendimethalin e alachlor, verificou-se que o aumento das doses proporcionou redução no número de plantas superior aos obtidos com trifluralin em todas as avaliações, sendo que aos 21 DAA e principalmente aos 28 DAA, a ausência das plantas em relação à testemunha sem aplicação foi, em média, superior a $80 \%$. 

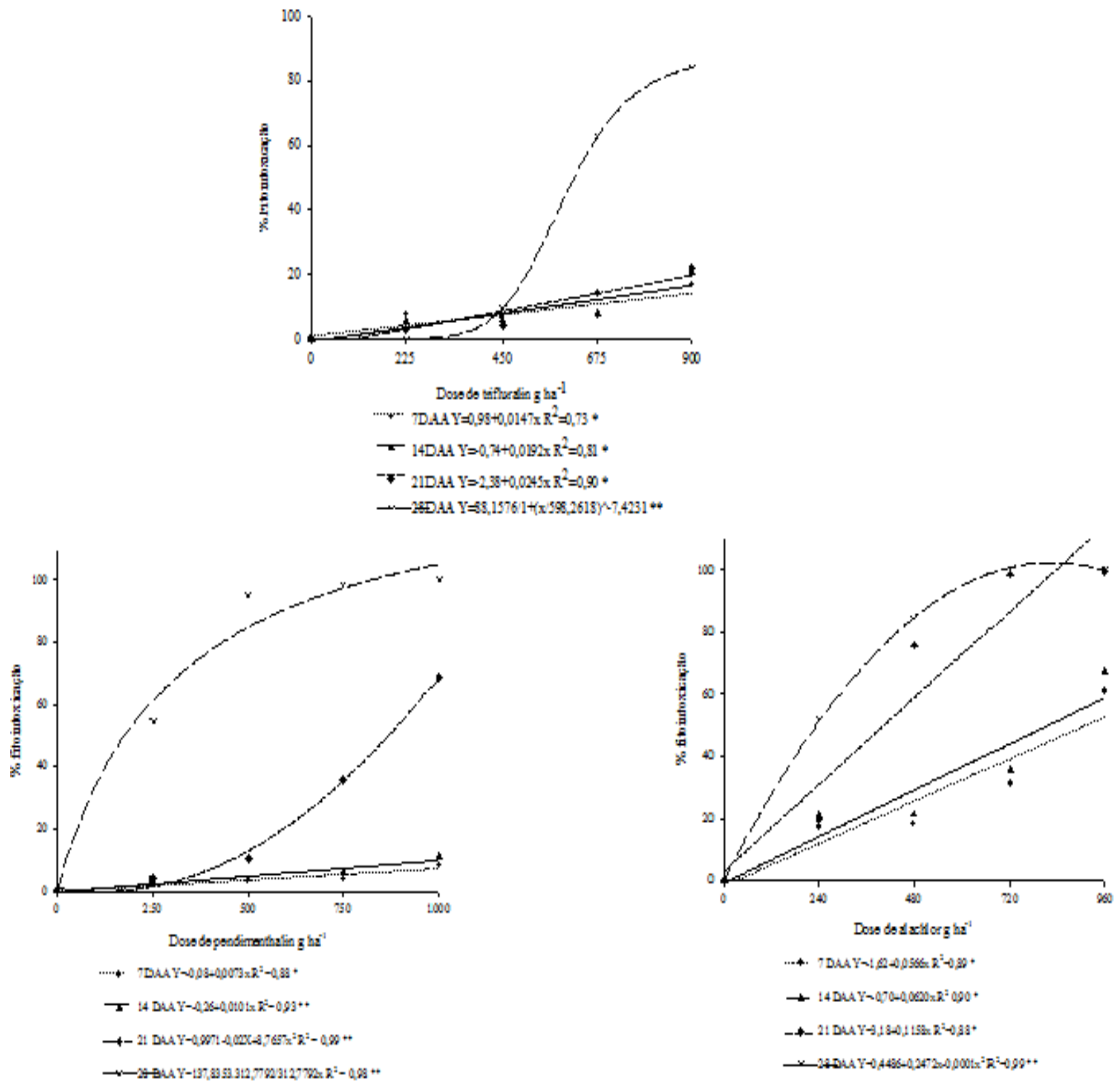

Figura 1. Fitointoxicação (\%) das plantas de crambe aos 7, 14, 21 e 28 DAA, submetidas à aplicação em pré-emergência dos herbicidas trifluralin (A), pendimethalin (B) e alachlor (C). 
A

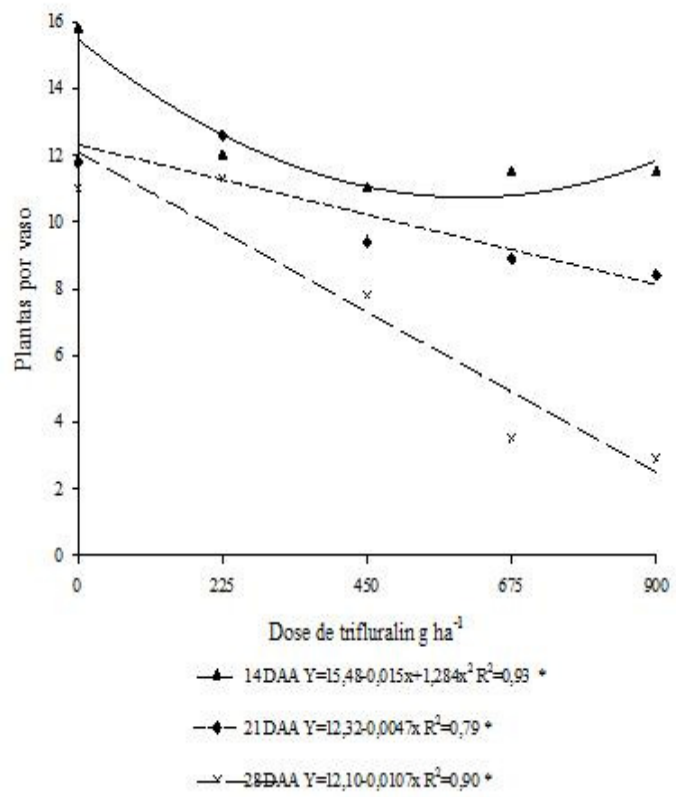

B
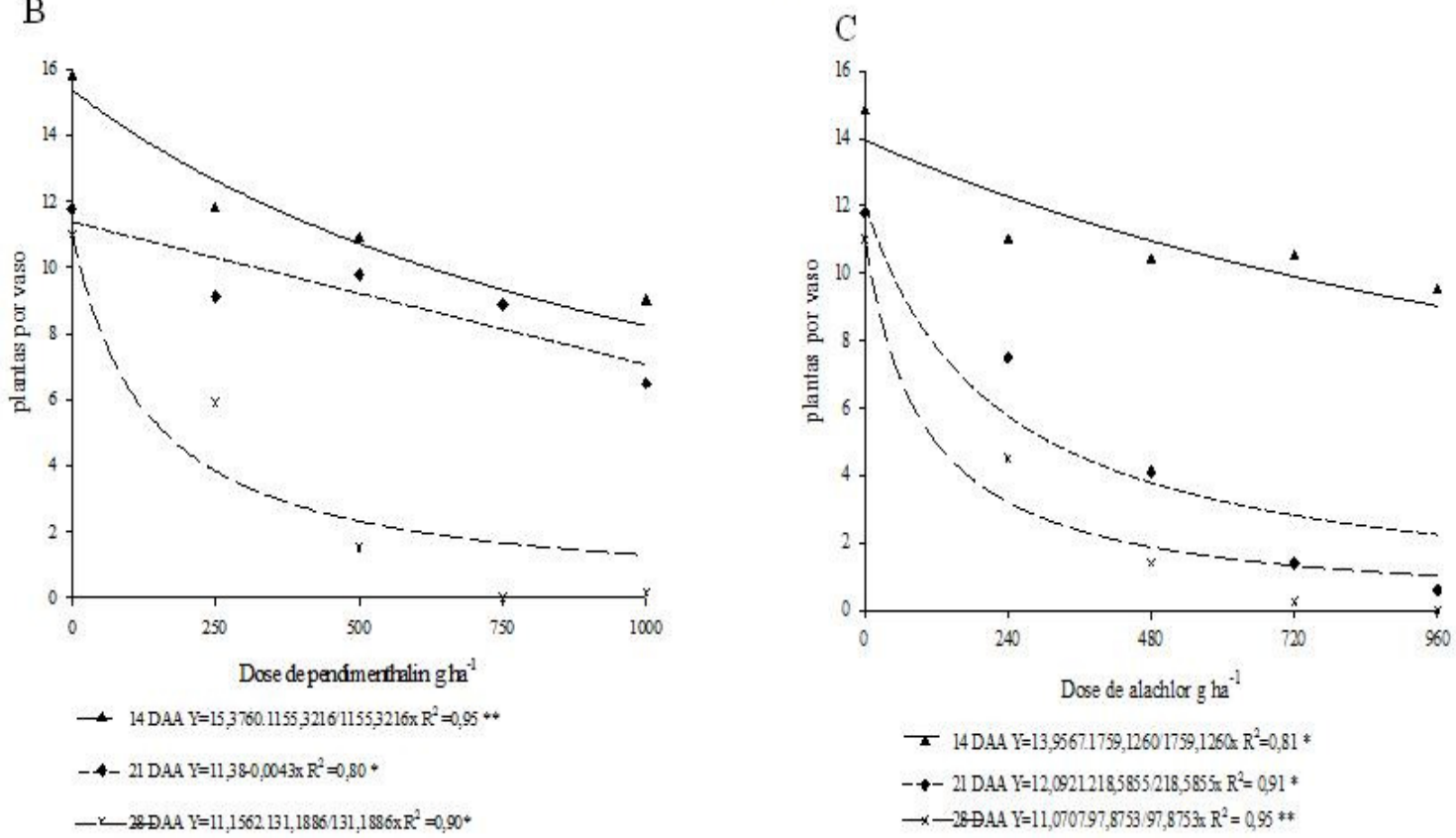

Figura 2. Densidade de plantas de crambe aos 7, 14, 21 e 28 DAA, submetidas à aplicação em pré-emergência dos herbicidas trifluralin (A), pendimethalin (B) e alachlor (C).

$\mathrm{Na}$ Tabela 1 estão representadas as pendimethalin + alachlor proporcionaram efeito porcentagens de fitointoxicação nas plantas de fitotóxico superiores a $60 \%$ a partir dos 7 DAA, crambe aos 7, 14, 21 e 28 DAA para as aumentando progressivamente a intensidade associações estudadas entre os herbicidas dos sintomas aos 14 e 21 DAA, e chegando a trifluralin, pendimethalin e alachlor. As atingir níveis de fitointoxicação da ordem de misturas em tanque de trifluralin + alachlor e $89,8 \%$ e $99,8 \%$, respectivamente, aos 28 DAA. 
Para trifluralin + pendimethalin, os sintomas de fitointoxicação foram significativamente pendimethalin e alachlor para desenvolvimento inferiores as demais misturas em tanque aos 7 , da cultura do crambe, uma vez que no estudo 14 e 21 DAA. Porém, as injúrias se tornaram utilizados isoladamente, os sintomas de mais severas aos 28 DAA $(96,4 \%)$, não intoxicação foram inferiores aos obtidos pelas diferindo significativamente das misturas misturas. Com isso constatou-se que nenhuma trifluralin + alachlor e pendimethalin + das associações estudadas em aplicação em préalachlor.

Esses resultados evidenciam efeito emergência foi seletiva à cultura do crambe.

negativo das associações entre trifluralin,

Tabela 1. Porcentagem de fitointoxicação nas plantas de crambe aos 7, 14, 21 e 28 DAA, submetidas à aplicação de misturas em tanque em pré-emergência.

\begin{tabular}{lccccc}
\hline \multirow{2}{*}{ Tratamentos } & \multirow{2}{*}{\begin{tabular}{c} 
Dose \\
\cline { 3 - 6 }
\end{tabular}} & \multicolumn{4}{c}{ \% de fitointoxicação } \\
\cline { 3 - 6 } & - & 0,0 & 0,0 & 0,0 & 0,0 \\
\hline Testemunha & $450+480$ & 60,8 & 64,9 & 92,1 & 89,8 \\
Trifluralin + alachlor & $480+500$ & 63,5 & 68,0 & 97,0 & 99,8 \\
Pendimethalin + alachlor & $450+500$ & 32,0 & 36,6 & 65,6 & 96,4 \\
Trifluralin + pendimethalin & & & &
\end{tabular}

DAA: Dias após a aplicação.

Dentre os herbicidas estudados apenas o trifluralin, em doses inferiores à $450 \mathrm{~g} \mathrm{ha}^{-1}$, apresentou potencial seletivo para $\mathrm{O}$ desenvolvimento inicial das plantas de crambe. Alachlor e pendimethalin, mesmo em doses baixas promovem alta fitointoxicação e redução acentuada no número de plantas, não sendo viáveis para cultura do crambe. As associações entre trifluralin, alachlor e pendimethalin em mistura em tanque não foram seletivas ao crambe.

Estes resultados preliminares evidenciam a necessidade de estudos mais abrangentes, que visem avaliar a seletividade de herbicidas à cultura do crambe.

\section{Referências}

BAEZ, O. Crambe a grande aposta das pesquisas no Mato Grosso do Sul. Pantanal News, 2007. Disponível em: $<$ http://www.biodieselbr.com/noticias/emfoco/crambe-a-grande-aposta-das-pesquisas-domato-grosso-do-sul.htm> Acesso em: 25 jan. 2011.
CAMPBELL, M.C.; WALTON, G. Alternative oilseed crops $x$ herbicide tolerance, 2008 Disponível em: $<$ http://www.agric.wa.gov.au/objtwr/imported_ assets/content/sust/biofuel/

clima_dafwa_alternativeoilseedsxherbicides_tri al1.pdf>. Acesso em 15 jan 2011.

CARNEIRO, S.M.T.P.G. et al. Ocorrência de Alternaria brassicicola em crambe (Crambe abyssinica) no estado do Paraná. Summa Phytopathology, v.35, n.2, p.154-155, 2009.

ENDRES, G.; SCHATZ, B. Crambe Production, 1993 Disponível em: $<$ http://www.ag.ndsu.edu/pubs/plantsci/crops/a 1010w.htm\#weed>. Acesso em 25 jan 2010.

FUNDAÇÃO MS. Crambe (Crambe abyssinica Hochst) uma opção rentável para a safrinha. Disponível em: $<$ http://www.fundacaoms.org.br>. Acesso em: 25 jan. 2011.

JASPER, S.P. et al. Análise energética da cultura do crambe (Crambe abyssinica Hochst) produzida em plantio direto. Engenharia Agrícola, v.30, n.3, p.395-403, 2010. 
OLIVEIRA JR., R.S. Mecanismo de ação de SILVA, A.A.; SILVA, J.F. Tópicos em herbicidas. In: OLIVEIRA Jr, R.S.; manejo de plantas daninhas. Viçosa, MG: CONSTANTIN, J. (Coord.).Plantas daninhas e UFV. 2007. 367 p.

seu manejo. Guaíba: Agropecuária, 2001. p. 209260.

SOCIEDADE BRASILEIRA DA CIÊNCIA

PITOL, C. Cultura do Crambe In:Tecnologia e DAS PLANTAS DANINHAS. Procedimentos Produção:Milho Safrinha e Culturas de Inverno. Fundação MS. p.85-88, 2008.

RODRIGUES, B.N.; ALMEIDA, F.S. Guia de herbicidas. $5^{\text {a }}$ edição. Londrina: Editora dos autores. 2005. 592p. para instalação, avaliação e análise de experimentos com herbicidas. Londrina: SBCPD, 1995. 42p.

STOUGAARD, R.N.; MOOMAW, R.S. Crambe (Crambe abyssinica) tolerance to herbicides. Weed Technology, v.5, n.2, p.566569, 1991. 